\title{
Improved Open Boundary Model for Plasma Particle Simulations
}

\author{
Hiroki HASEGAWA ${ }^{\text {a) }}$ and Tetsuya SATO ${ }^{1)}$ \\ Japan Agency for Marine-Earth Science and Technology (JAMSTEC), Yokohama 236-0001, Japan \\ 1) University of Hyogo, Kobe 650-0044, Japan \\ (Received 15 March 2010 / Accepted 9 April 2010)
}

\begin{abstract}
An open system one-dimensional electrostatic particle code that adopts the new constant current generator model has been developed. Using this new model, we have been able to study various phenomena at large electron drift velocities, where the original model is not applicable. In this new model, the contribution of the ion flux, which is not considered in the original model, is added into the electric current. By examining the code, we find that the result in a case where the electron drift velocity is smaller than the electron thermal velocity is similar to that of a previous study, which showed double layer creation as a result of ion-acoustic instability. We also present the results of simulations of an electron drift velocity comparable to or larger than the electron thermal velocity.
\end{abstract}

(c) 2010 The Japan Society of Plasma Science and Nuclear Fusion Research

Keywords: particle simulation, open system, double layer, electron acceleration, auroral energetic electron

DOI: $10.1585 /$ pfr.5.020

\section{Introduction}

A number of plasma particle simulations adopted periodic or reflective boundary conditions for particles. Thus, their electric charge neutrality can be rigidly maintained. However, those boundary conditions strictly confine calculable physical phenomena. For instance, although spontaneous production of ion-acoustic double layers was demonstrated by a one-dimensional electrostatic particle simulation under a periodic boundary condition [1], the electric potential difference of an observed double layer is comparable to just the electron thermal energy. This is because electrons accelerated by the double layers are ejected from the downstream boundary and then become incoming electrons from the upstream boundary. The existence of those "dirty" electrons prevents long-term evolution of the system. Another reason is that a closed system has only the initial free energy and cannot be provided with additional free energy from external sources.

Therefore, Sato et al. [2] developed an open system one-dimensional code that adopts the constant current generator model [3]. Using that code they showed that super ion-acoustic double layers, the electric potential difference of which can exceed ten times the electron thermal energy, are formed in an open system in which a constant electron flow is continuously supplied. A difficulty in code development is the consideration of incoming electrons from the downstream boundary because of the small electron drift velocity, $v_{\mathrm{d}} \lesssim v_{\mathrm{Te}}$, where $v_{\mathrm{Te}}$ is the electron thermal velocity.

Furthermore, this open boundary model was extended to a two-dimensional electrostatic code. A V-shaped dc po-

author's e-mail: hasegawa.hiroki@nifs.ac.jp

a) Now at National Institute for Fusion Science, Toki, Japan. tential structure created by the current-driven ion-cyclotron instability was demonstrated [4]. This feature had not been found under a circulating boundary condition [5].

Potential structures similar to the super ion-acoustic double layer or the V-shaped structure are observed in auroral energetic electron acceleration regions [6-9], where a hot electron flux with a drift velocity comparable to (or smaller than) the electron thermal velocity is expected to be supplied from the plasma sheet. Thus, those simulation studies in which the constant current generator model is adopted are thought to explain microscopic processes occurring in auroral electron production regions.

On the other hand, a recent study reported that a microscopic effect of auroral energetic electrons plays an important role in forming the macroscopic structure of the quiet auroral arc [10]. In that study, the effect of auroral energetic electrons was investigated with a holistic simulation that was successfully performed on the basis of the macro-micro interlocked (MMI) algorithm [11]. The holistic simulation code consists of a three-dimensional magnetohydrodynamic (MHD) code for a dipole magnetosphereionosphere coupling system and a one-dimensional electrostatic plasma particle code for auroral energetic electrons production by ion-acoustic double layers. The MHD and particle codes are connected as follows. First, a maximum value of the field-aligned current in an intensive growth area is transferred from MHD (macroscopic) side to the particle (microscopic) side. Next, on the particle side, the electron drift velocity $v_{\mathrm{d}}$ is calculated from the maximum value of the field-aligned current. Then, the particle simulation is executed using $v_{\mathrm{d}}$ as the initial condition. At the end of the particle simulation, the rate of ion production owing to precipitating electrons in the ionosphere 
is computed from the energy spectrum of electrons on the downstream boundary of the system. Finally, the obtained ion production rate is given to the MHD side. Because this procedure is used, the particle simulation code on the MMI simulation must be able to treat any value of the electron drift velocity.

However, when $v_{\mathrm{d}} \gtrsim v_{\mathrm{Te}}$, calculations by a code that adopts the original constant current generator model falter frequently. Although Takamaru et al. [3] showed that test runs up to one plasma period are successfully carried out at $v_{\mathrm{d}} \sim v_{\mathrm{Te}}$ when about $10^{4}$ particles per grid are prepared, executions running long enough to create a super double layer often fail.

In the present study, we develop an improved open boundary model whose computations are free from the above problem and a one-dimensional electrostatic code that adopts this new model and is applicable to the auroral MMI simulation code. In Sec. 2, we review the original constant current generator model and describe the improved model. In Sec. 3, we describe the particle simulation parameters and the procedure for the auroral MMI code and show the results of test runs. In Sec. 4, we summarize our work.

\section{Methodology}

\subsection{Open boundary system connected to a constant current generator}

We assume that the initial velocity distribution of electrons in a system is supplied by the shifted Maxwellian having a drift velocity $v_{\mathrm{d}}$,

$$
f_{\mathrm{e}}^{\mathrm{ini}}(v)=\frac{1}{\sqrt{2 \pi v_{\mathrm{Te}}^{2}}} \exp \left[-\frac{\left(v-v_{\mathrm{d}}\right)^{2}}{2 v_{\mathrm{Te}}^{2}}\right],
$$

and that of ions is assumed to be given by the Maxwellian,

$$
f_{\mathrm{i}}^{\mathrm{ini}}(v)=\frac{1}{\sqrt{2 \pi v_{\mathrm{Ti}}^{2}}} \exp \left(-\frac{v^{2}}{2 v_{\mathrm{Ti}}^{2}}\right),
$$

where $v_{\mathrm{Ti}}$ is the ion thermal velocity. The particle fluxes at the upstream and downstream boundaries, respectively, are given by

$$
\begin{aligned}
& F_{s}^{\text {up }}=F_{s, \text { in }}^{\text {up }}+F_{s, \text { out }}^{\text {up }}, \\
& F_{s}^{\text {down }}=F_{s, \text { out }}^{\text {down }}+F_{s, \text { in }}^{\text {down }},
\end{aligned}
$$

where

$$
\begin{aligned}
& F_{s, \text { in }}^{\text {up }}=n_{s}^{\text {up }} \int_{0}^{+\infty} v f_{s}^{\text {up }}(v) \mathrm{d} v, \\
& F_{s, \text { out }}^{\text {up }}=n_{s}^{\text {up }} \int_{-\infty}^{0} v f_{s}^{\text {up }}(v) \mathrm{d} v, \\
& F_{s, \text { out }}^{\text {down }}=n_{s}^{\text {down }} \int_{0}^{+\infty} v f_{s}^{\text {down }}(v) \mathrm{d} v,
\end{aligned}
$$

$$
F_{s, \text { in }}^{\text {down }}=n_{s}^{\text {down }} \int_{-\infty}^{0} v f_{s}^{\text {down }}(v) \mathrm{d} v
$$

Here, $n$ is the average number density of particles. The superscripts "up" and "down" refer to the upstream and downstream boundaries, respectively. The subscript $s$ represents electrons (e) or ions (i). The subscripts "in" and "out" refer to the incoming direction into the system and the outgoing direction from the system, respectively.

Using these fluxes, we obtain the numbers of particles passing the boundaries during the finite time step $\Delta t$,

$$
\begin{gathered}
N_{s, \text { in }}^{\text {up }}=\left(n_{s}^{\text {up }} \lambda_{\mathrm{D}}\right)\left(\omega_{\mathrm{pe}} \Delta t\right) \\
\times \int_{0}^{+\infty} \frac{v}{v_{\mathrm{Te}}} f_{s}^{\text {up }}(v) \mathrm{d} v, \\
N_{s, \text { out }}^{\text {up }}=-\left(n_{s}^{\text {up }} \lambda_{\mathrm{D}}\right)\left(\omega_{\mathrm{pe}} \Delta t\right) \\
\times \int_{-\infty}^{0} \frac{v}{v_{\mathrm{Te}}} f_{s}^{\text {up }}(v) \mathrm{d} v, \\
N_{s, \text { out }}^{\text {down }}=\left(n_{s}^{\text {down }} \lambda_{\mathrm{D}}\right)\left(\omega_{\mathrm{pe}} \Delta t\right) \\
\quad \times \int_{0}^{+\infty} \frac{v}{v_{\mathrm{Te}}} f_{s}^{\text {down }}(v) \mathrm{d} v, \\
N_{s, \text { in }}^{\text {down }}=-\left(n_{s}^{\text {down }} \lambda_{\mathrm{D}}\right)\left(\omega_{\mathrm{pe}} \Delta t\right) \\
\quad \times \int_{-\infty}^{0} \frac{v}{v_{\mathrm{Te}}} f_{s}^{\text {down }}(v) \mathrm{d} v,
\end{gathered}
$$

where $\lambda_{\mathrm{D}}$ and $\omega_{\mathrm{pe}}$ are the Debye length and plasma frequency, respectively.

In the constant current generator model, the electric current produced by the electron flux at the upstream boundary is assumed to be constant and equal to that at the downstream boundary, i.e.,

$$
F_{\mathrm{e}}^{\text {up }}=F_{\mathrm{e}}^{\text {down }}=\text { const. }
$$

This condition is equivalent to

$$
N_{\mathrm{e}, \text { net }}^{\mathrm{up}}=N_{\mathrm{e}, \text { net }}^{\text {down }} \equiv N_{\mathrm{e}, \text { net }}=\text { const., }
$$

where

$$
\begin{aligned}
& N_{\mathrm{e}, \text { net }}^{\text {up }}=N_{\mathrm{e}, \text { in }}^{\text {up }}-N_{\mathrm{e}, \text { out }}^{\text {up }}, \\
& N_{\mathrm{e}, \text { net }}^{\text {down }}=N_{\mathrm{e}, \text { out }}^{\text {down }}-N_{\mathrm{e}, \text { in }}^{\text {down }} .
\end{aligned}
$$

Accordingly, the numbers of injected electrons at the upstream and the downstream boundaries are given by

$$
\begin{aligned}
& N_{\mathrm{e}, \text { in }}^{\mathrm{up}}=N_{\mathrm{e}, \text { net }}+N_{\mathrm{e}, \text { out }}^{\mathrm{up}}, \\
& N_{\mathrm{e}, \text { in }}^{\text {down }}=N_{\mathrm{e}, \text { out }}^{\text {down }}-N_{\mathrm{e}, \text { net }},
\end{aligned}
$$

respectively.

In actual computation, these numbers are calculated at every step. Then, the injected particles $\left(N_{\mathrm{e}, \text { in }}^{\mathrm{up}}\right.$ and $\left.N_{\mathrm{e}, \mathrm{in}}^{\mathrm{down}}\right)$ 
are distributed in the velocity space by the cumulative distribution function derived from $f_{\mathrm{e}}^{\mathrm{ini}}(v)$. The initial positions of the injected particles are set as $x_{j 0}=R_{j} v_{j} \Delta t+x_{\text {up } \text { or down }}$, where the subscript $j$ refers to the particle number, and $R$ is a random number.

With this model, the total number of electrons in the system is conserved because

$$
\frac{\partial N_{\mathrm{e}}^{\text {total }}}{\partial t}=F_{\mathrm{e}}^{\mathrm{up}}-F_{\mathrm{e}}^{\mathrm{down}}=0 .
$$

On the other hand, the ion fluxes at the upstream and downstream boundaries are assumed to satisfy $F_{\mathrm{i}}^{\text {up }}=$ $F_{\mathrm{i}}^{\text {down }}=0$. Thus, the numbers of injected ions at the upstream and downstream boundaries are given by

$$
\begin{aligned}
& N_{\mathrm{i}, \text { in }}^{\text {up }}=N_{\mathrm{i}, \text { out }}^{\text {up }}, \\
& N_{\mathrm{i}, \text { in }}^{\text {down }}=N_{\mathrm{i}, \text { out }}^{\text {down }},
\end{aligned}
$$

respectively.

From Eq. (18), this model is applicable when $N_{\mathrm{e}, \text { out }}^{\text {down }}>$ $N_{\text {e,net }}$. Takamaru et al. [3] investigated the range of applicability by test runs up to one plasma period and showed that the number of simulation particles per grid should be comparable to (or larger than) $10^{4}$ to satisfy this condition. However, even if more than $10^{4}$ particles per grid are prepared, $N_{\mathrm{e}, \text { out }}^{\text {down }}$ frequently becomes smaller than $N_{\mathrm{e}, \text { net }}$ at $v_{\mathrm{d}} \gtrsim v_{\mathrm{Te}}$ during an execution long enough to create a super double layer. This is because a number of electrons stay in the system.

\subsection{Improved open boundary model}

Since the value of the field-aligned current, i.e., the electron drift velocity transferred from the MHD code, cannot be anticipated, the open system particle simulation code in the MMI simulation should be able to treat any $v_{\mathrm{d}}$ even one larger than $v_{\mathrm{Te}}$. Thus, to install the open system particle code in the MMI code, a new open boundary model that avoids this problem, which was demonstrated in the previous subsection, must be developed. For this purpose, although the original current generator model assumes that the electric current consists of only the electron flux, the contribution of the ion flux is also considered in the new model.

The electric currents at the upstream and downstream boundaries are given by

$$
\begin{aligned}
& J^{\text {up }}=q_{\mathrm{i}} F_{\mathrm{i}}^{\text {up }}+q_{\mathrm{e}} F_{\mathrm{e}}^{\text {up }}, \\
& J^{\text {down }}=q_{\mathrm{i}} F_{\mathrm{i}}^{\text {down }}+q_{\mathrm{e}} F_{\mathrm{e}}^{\text {down }},
\end{aligned}
$$

where $q$ is the electric charge of a particle. Here, we suppose that the current at each boundary is constant and that the two currents are equal, i.e.,

$$
J^{\text {up }}=J^{\text {down }}=\text { const. }
$$

This condition is equivalent to

$$
N_{\text {net }}^{\text {up }}=N_{\text {net }}^{\text {down }} \equiv N_{\text {net }}=\text { const., }
$$

where

$$
\begin{aligned}
N_{\text {net }}^{\text {up }}= & -J^{\text {up }} \Delta t / q_{\mathrm{i}} \\
= & \left(N_{\mathrm{e}, \text { in }}^{\text {up }}-N_{\mathrm{e}, \text { out }}^{\text {up }}\right)-\left(N_{\mathrm{i}, \text { in }}^{\text {up }}-N_{\mathrm{i}, \text { out }}^{\text {up }}\right), \\
N_{\text {net }}^{\text {down }}= & -J^{\text {down }} \Delta t / q_{\mathrm{i}} \\
= & \left(N_{\mathrm{e}, \text { out }}^{\text {down }}-N_{\mathrm{e}, \text { in }}^{\text {down }}\right) \\
& -\left(N_{\mathrm{i}, \text { out }}^{\text {down }}-N_{\mathrm{i}, \text { in }}^{\text {down }}\right) .
\end{aligned}
$$

It is assumed that $q_{\mathrm{i}}=-q_{\mathrm{e}}(>0)$.

If $N_{\mathrm{e}, \text { out }}^{\text {down }} \geqq N_{\text {net }}$, the numbers of injected particles are obtained as shown in the previous subsection.

On the other hand, when $N_{\mathrm{e}, \text { out }}^{\text {down }}<N_{\text {net }}$, we assume that the number of injected electrons at the downstream boundary is zero,

$$
N_{\mathrm{e}, \text { in }}^{\text {down }}=0 .
$$

Further, the numbers of injected electrons and ions at the upstream boundary, $N_{\mathrm{e}, \text { in }}^{\mathrm{up}}$ and $N_{\mathrm{i}, \text { in }}^{\mathrm{up}}$, are given by Eqs. (17) and (20), respectively. Accordingly, the number of injected ions at the downstream boundary is

$$
N_{\mathrm{i}, \text { in }}^{\text {down }}=N_{\mathrm{i}, \text { out }}^{\text {down }}+\left(N_{\text {net }}-N_{\mathrm{e}, \text { out }}^{\text {down }}\right) .
$$

In this case, the total number of both electrons and ions in the system increases by $N_{\text {net }}-N_{\mathrm{e} \text {,out }}^{\text {down }}$. Thus, memory for these additional particles must be prepared in the code. Although the total number of particles in the system is not conserved, the electric charge neutrality $\left(\rho^{\text {total }}=0\right)$ is still satisfied because

$$
\frac{\partial \rho^{\text {total }}}{\partial t}=J^{\text {up }}-J^{\text {down }}=0 .
$$

In this new model, the injected particles are also distributed in the velocity space by the cumulative distribution function. The initial positions of the injected particles are given as shown in the previous section. However, the cumulative distribution function at the downstream boundary is derived from the velocity distribution function observed at the downstream edge.

\section{Examination of the Code}

\subsection{Procedure and parameters}

To obtain the rate of ion production due to precipitating electrons in the ionosphere, the auroral MMI simulation code computes the microscopic process of electron acceleration by the ion-acoustic double layer using a onedimensional electrostatic code that adopts the new open boundary model. In this paper, we examine the particle simulation code installed in the auroral MMI simulation code.

The simulation parameters are as follows. The total system length is $L=1024 \lambda_{\mathrm{D}}$. The grid spacing is $\Delta_{\mathrm{g}}=\lambda_{\mathrm{D}}$. The ion-to-electron mass ratio is $m_{\mathrm{i}} / m_{\mathrm{e}}=100$. The electron-to-ion temperature ratio is $T_{\mathrm{e}} / T_{\mathrm{i}}=20$. There 
are 10,240 electrons and an equal number of ions per grid. The total number of time steps for one run is fixed at $N_{\mathrm{t}}=39,250$ to make the real computation time of runs flat.

In this particle simulation, the potential difference must grow enough to accelerate electrons. At $v_{\mathrm{d}} \lesssim v_{\mathrm{Te}}$, double layers are created by ion-acoustic instability. Thus, runs must be continued for at least $1000 \tau_{\text {pe }}$ [2], where $\tau_{\mathrm{pe}}=2 \pi / \omega_{\mathrm{pe}}$. On the other hand, when $v_{\mathrm{d}} \gg v_{\mathrm{Te}}$, the physical process probably consists of phenomena arising from two-stream (Bunemann) instability. Therefore, potential structures are thought to form for a short time $\left(\lesssim \omega_{\mathrm{pe}}^{-1}\left(m_{\mathrm{i}} / m_{\mathrm{e}}\right)^{1 / 2}\left(\lambda / \lambda_{\mathrm{D}}\right) /\left(v_{\mathrm{d}} / v_{\mathrm{Te}}\right)\right)$, where $\lambda$ is the characteristic length of a wave. From this estimation, the time step is given by

$$
\Delta t=\left\{\begin{array}{lr}
\Delta t_{0} & \left(v_{\mathrm{d}} \leqq v_{\mathrm{c}}\right), \\
\frac{v_{\mathrm{c}}}{v_{\mathrm{d}}}\left[\Delta t_{0}+\left(\frac{L}{N_{\mathrm{t}} v_{\mathrm{c}}}-\Delta t_{0}\right)\right. & \left.\frac{v_{\mathrm{d}}-v_{\mathrm{c}}}{v_{\mathrm{u}}-v_{\mathrm{c}}}\right] \\
\left(v_{\mathrm{c}}<v_{\mathrm{d}} \leqq v_{\mathrm{u}}\right), \\
\frac{L}{N_{\mathrm{t}} v_{\mathrm{d}}} & \left(v_{\mathrm{u}}<v_{\mathrm{d}}\right),
\end{array}\right.
$$

where $\Delta t_{0}=0.16 \omega_{\mathrm{pe}}^{-1}, v_{\mathrm{c}} / v_{\mathrm{Te}}=1.0$, and $v_{\mathrm{u}} / v_{\mathrm{Te}}=2.0$.

To execute the MMI code efficiently, the computation speed of the microscopic side should be much faster than that of the macroscopic side. Thus, the particle code is parallelized by the message-passing interface (MPI), a distributed parallel processing language [12]. Furthermore, we adopt the parallelized Mersenne Twister method $[13,14]$ for random number creation at particle injection.

\subsection{Simulation results}

Figures 1 and 2 show the result of a simulation for $v_{\mathrm{d}}=0.7 v_{\mathrm{Te}}$. The top, middle, and bottom panels present the spatial profile of electric potential and distributions of electrons and ions in space-velocity phase space, respectively. At $t=160 \tau_{\text {pe }}$, as shown in Fig. 1, stair-like weak double layers on the order of the electron thermal energy arise. Such a structure is usually observed at an early stage [2]. In the electron phase space plot, electron acceleration in the positive direction is found in the region where each double layer appears. An electron void also appears on the downstream side of each double layer. The ion phase space plot shows ion acceleration in the negative direction. On the other hand, at $t=880 \tau_{\text {pe }}$, as shown in Fig. 2, a shocklike structure with a potential difference of about 40 times the electron thermal energy appears around $x=300 \lambda_{\mathrm{D}}$. The phase space plots show that this super ion-acoustic double layer strongly accelerates the forward-moving electrons and the backward-moving ions. The result shown here indicates that the code reproduces well the result of a previous study [2], demonstrating that the code is valid.

Figures 3 and 4 show the result of a simulation for
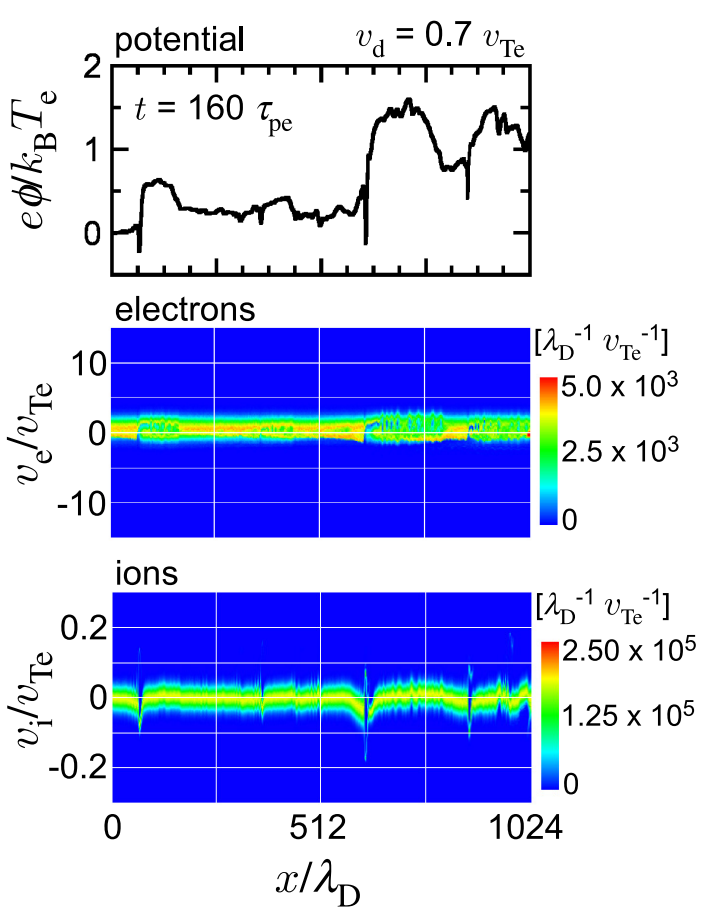

Fig. 1 Spatial profile of the electric potential averaged over $10 \tau_{\mathrm{pe}}$ at $t=160 \tau_{\mathrm{pe}}$. Distributions of electrons and ions in space-velocity phase space are plotted in color in the middle and bottom panels, respectively. The electron drift velocity is $v_{\mathrm{d}}=0.7 v_{\mathrm{Te}}$.
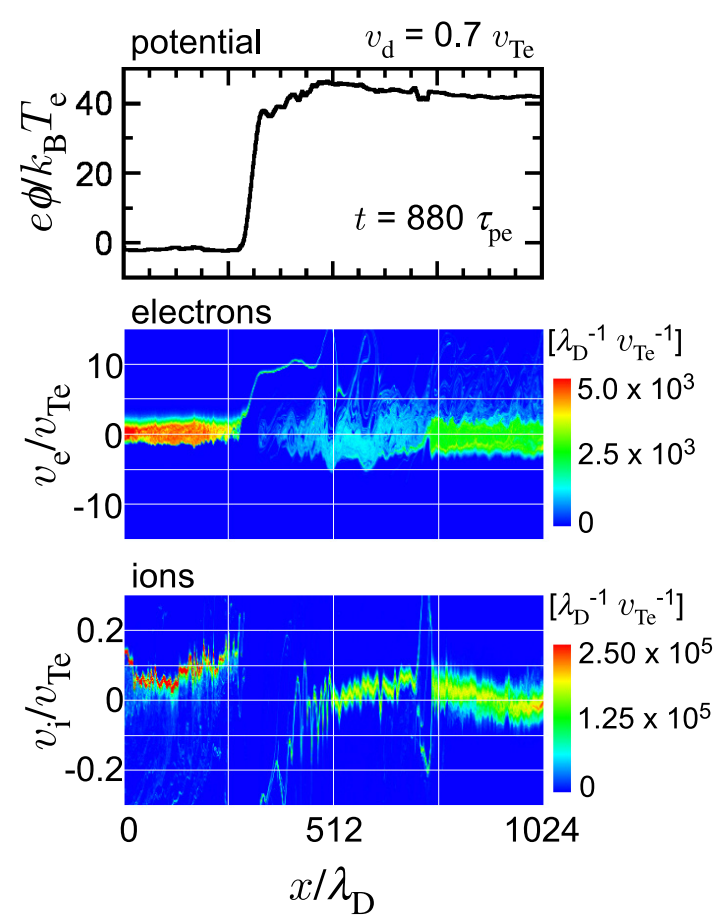

Fig. 2 Spatial profile of the electric potential and the distributions of electrons and ions in the space-velocity phase space at $t=880 \tau_{\mathrm{pe}}$; the electron drift velocity is $v_{\mathrm{d}}=$ $0.7 v_{\text {Te }}$. 

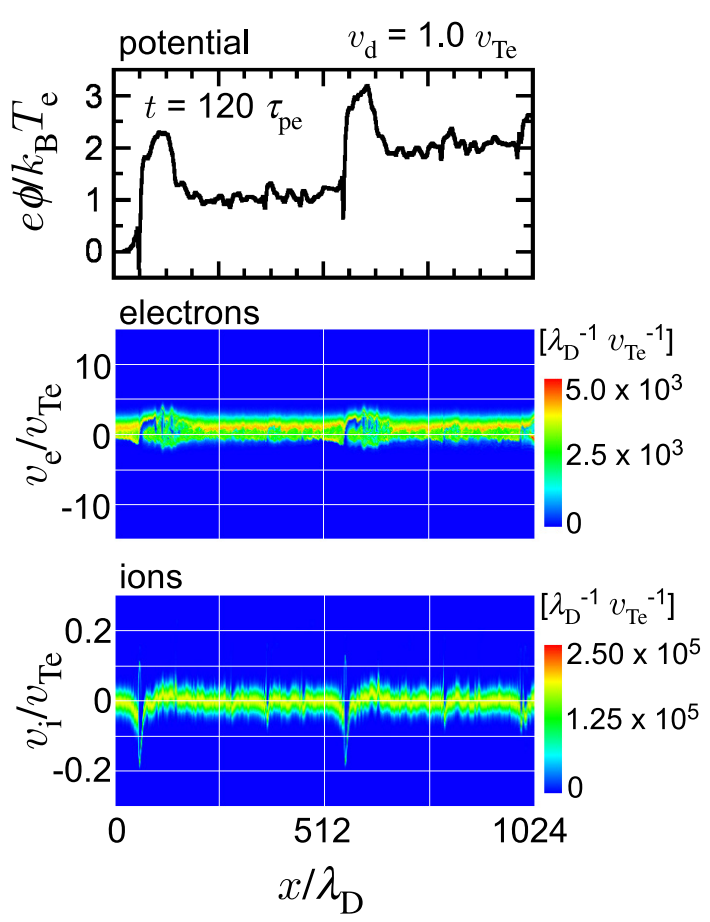

Fig. 3 Spatial profile of the electric potential averaged over $10 \tau_{\mathrm{pe}}$ at $t=120 \tau_{\mathrm{pe}}$. Distributions of electrons and ions in the space-velocity phase space are plotted in color in the middle and bottom panels, respectively. The electron drift velocity is $v_{\mathrm{d}}=1.0 v_{\mathrm{Te}}$.
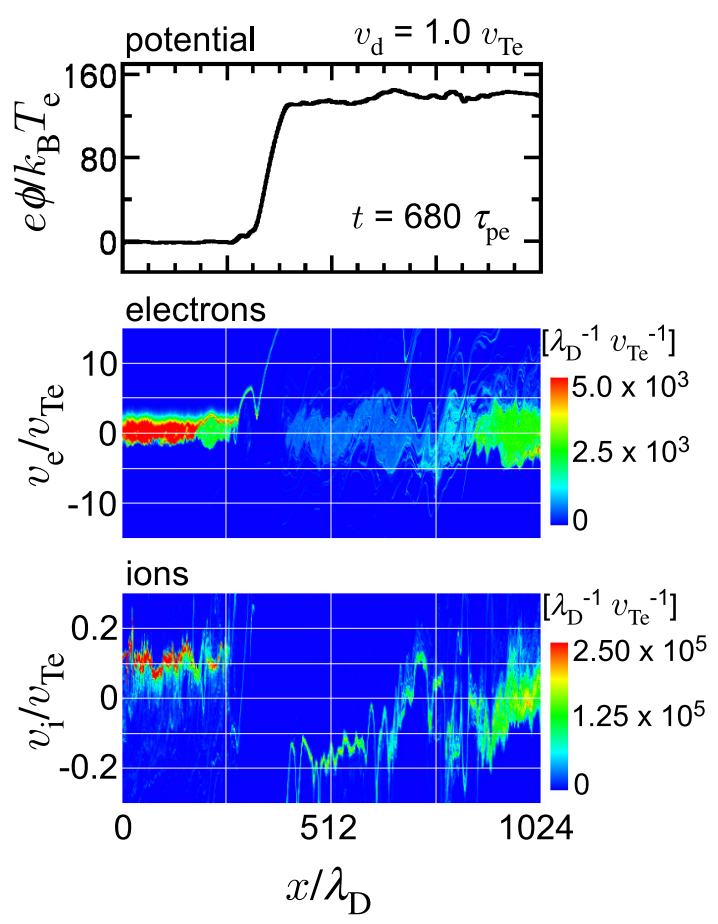

Fig. 4 Spatial profile of the electric potential and the distributions of electrons and ions in the space-velocity phase space at $t=680 \tau_{\mathrm{pe}}$; electron drift velocity is $v_{\mathrm{d}}=1.0 v_{\mathrm{Te}}$.
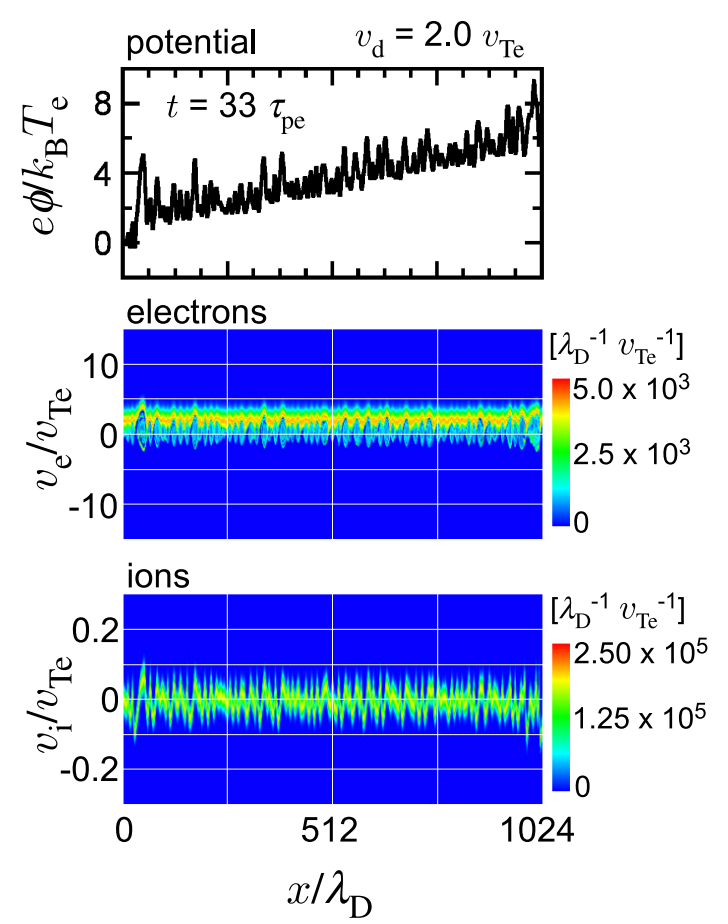

Fig. 5 Spatial profile of the electric potential averaged over $0.8 \tau_{\mathrm{pe}}$ at $t=33 \tau_{\mathrm{pe}}$. Distributions of electrons and ions in the space-velocity phase space are plotted in color in the middle and bottom panels, respectively. Electron drift velocity is $v_{\mathrm{d}}=2.0 v_{\mathrm{Te}}$.
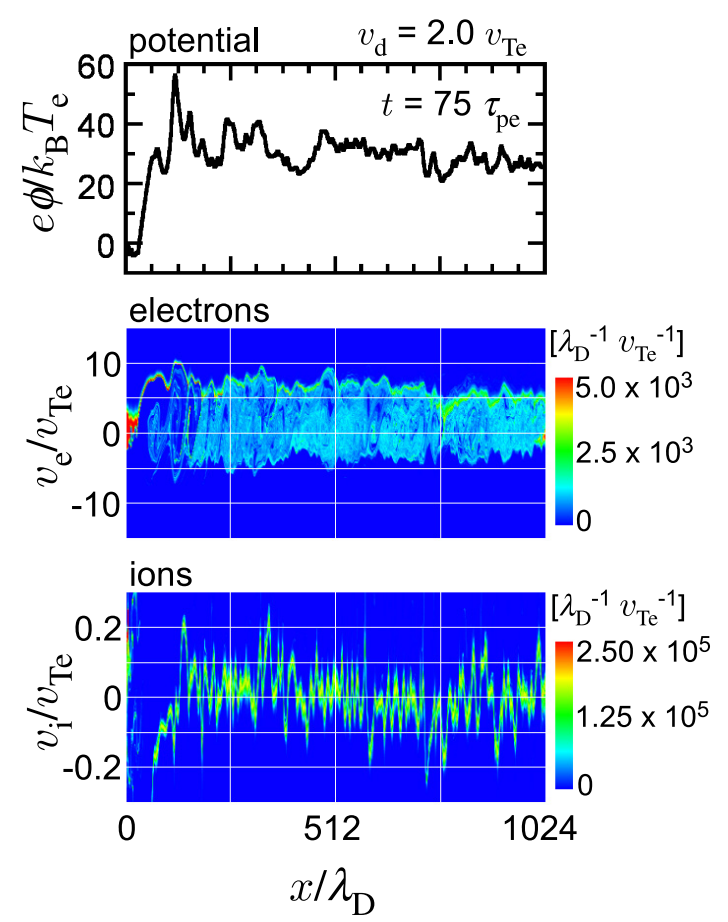

Fig. 6 Spatial profile of the electric potential and the distributions of electrons and ions in the space-velocity phase space at $t=75 \tau_{\mathrm{pe}}$; electron drift velocity is $v_{\mathrm{d}}=2.0 v_{\mathrm{Te}}$. 


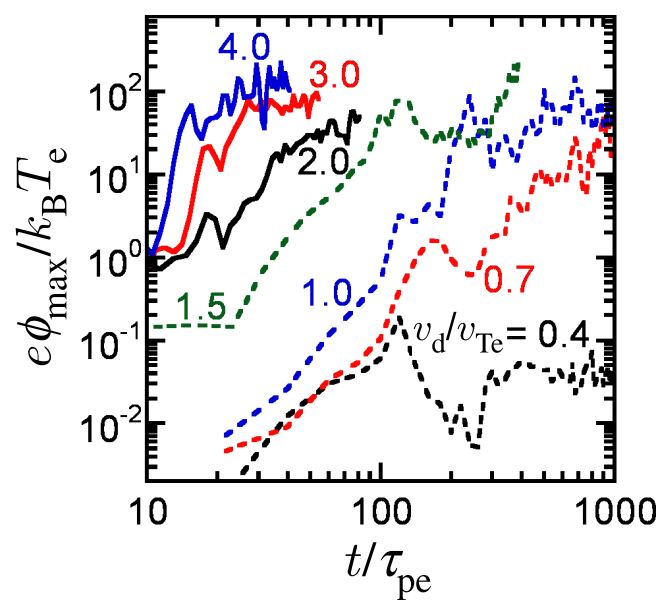

Fig. 7 Time variations of the electric potential maxima for $v_{\mathrm{d}} / v_{\mathrm{Te}}=0.4$ (black dashed line), 0.7 (red dashed line), 1.0 (blue dashed line), 1.5 (green dashed line), 2.0 (black solid line), 3.0 (red solid line), and 4.0 (blue solid line). Horizontal axis (time axis) is on a logarithmic scale.

$v_{\mathrm{d}}=1.0 v_{\mathrm{Te}}$. Figure 3 shows that stair-like weak double layers also appear at an early stage when $v_{\mathrm{d}}=1.0 v_{\mathrm{Te}}$. Furthermore, after a while, a super ion-acoustic double layer with a potential amplitude of about 120 times the electron thermal energy arises around $x=350 \lambda_{\mathrm{D}}$, as shown in Fig. 4. Thus, it may be concluded that double layer creation by ion-acoustic instability also occurs in the vicinity of $v_{\mathrm{d}} \sim v_{\mathrm{Te}}$.

Figures 5 and 6 present the result of a simulation in which $v_{\mathrm{d}}=2.0 v_{\mathrm{Te}}$. Figure 5 shows the profiles at an early stage $\left(t=33 \tau_{\mathrm{pe}}\right)$. The middle panel shows that some electrons are bunched and electron vortices are formed. Also, the bottom panel shows that the ion distribution is modified by local charge neutrality violation, which is the result of the electron motion. These features are thought to be produced by the Bunemann instability. After a while, a potential structure with an amplitude comparable with that of the super ion-acoustic double layer is created, as shown in Fig. 6. These facts indicate that a double layer is formed as a result of the Bunemann instability in the case of $v_{\mathrm{d}}=2.0 v_{\mathrm{Te}}$.

In Fig. 7, we show the time variations of the potential maxima for $v_{\mathrm{d}} / v_{\mathrm{Te}}=0.4,0.7,1.0,1.5,2.0,3.0$, and 4.0. At $v_{\mathrm{d}} / v_{\mathrm{Te}}=0.4$, the growth of the potential is negligible. However, when the electron drift velocity is larger than $0.7 v_{\mathrm{Te}}$, the potential amplitude increases to about 100 times the electron thermal energy by the formation of the super ion-acoustic double layer, which requires at least $100 \tau_{\text {pe }}$. Figure 7 also indicates that the saturated amplitude increases with the drift velocity.

On the other hand, when $v_{\mathrm{d}} \gtrsim 2.0 v_{\mathrm{Te}}$, a huge potential difference is formed by the Bunemann instability. Figure 7 shows that the time required for double layer production is smaller than $100 \tau_{\mathrm{pe}}$. In this case, the saturated amplitude
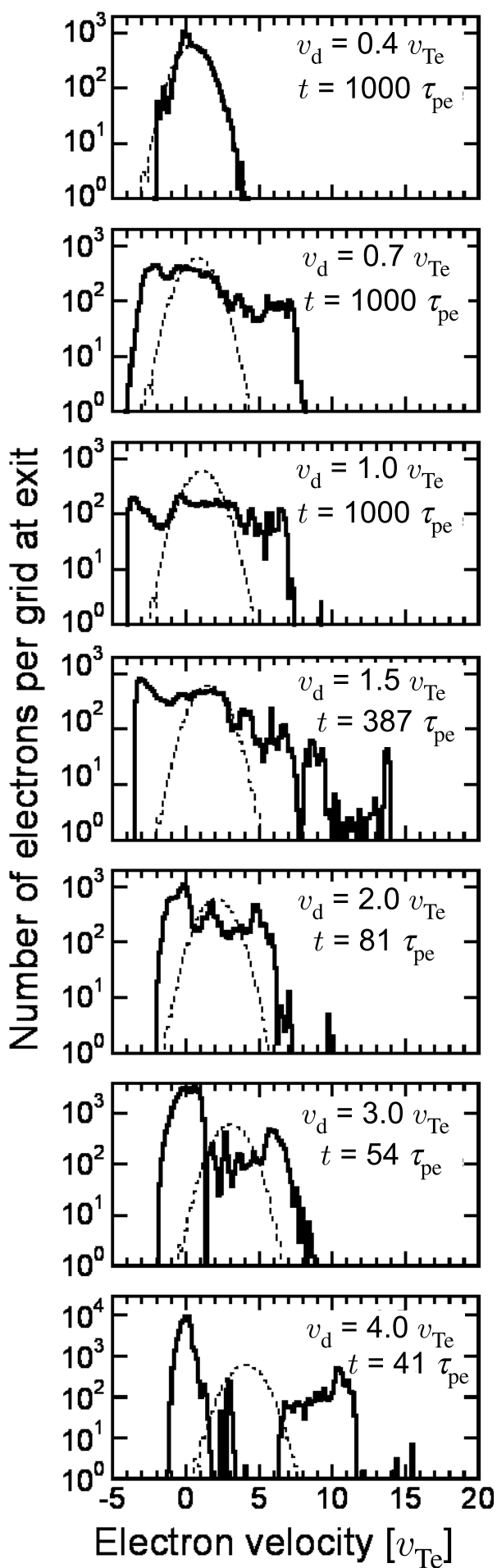

Fig. 8 Velocity distributions of electrons around the downstream edge at the end of the simulations for $v_{\mathrm{d}} / v_{\mathrm{Te}}=0.4,0.7$, 1.0, 1.5, 2.0, 3.0, and 4.0. The dashed line in each panel represents the initial distribution.

also increases with the drift velocity.

Figure 8 shows the electron velocity distributions around the downstream edge at the end of the simulations for $v_{\mathrm{d}} / v_{\mathrm{Te}}=0.4,0.7,1.0,1.5,2.0,3.0$, and 4.0. At $v_{\mathrm{d}} / v_{\mathrm{Te}}=0.4$, few electrons are accelerated, since double 
layers are not formed. However, when $v_{\mathrm{d}} \gtrsim 0.7 v_{\mathrm{Te}}$, a number of electrons are accelerated by double layers. If the electron thermal energy is assumed to be $100 \mathrm{eV}$, the energy of accelerated electrons reaches to $1 \sim 10 \mathrm{keV}$. This is comparable with that of observed auroral energetic electrons [15-18].

\section{Summary}

We developed an open system one-dimensional electrostatic particle code that adopts the new constant current generator model. Using this new model, we have been able to compute phenomena under large electron drift velocity conditions $\left(v_{\mathrm{d}} \gtrsim v_{\mathrm{Te}}\right)$, though the original model is not applicable in such a situation. The difference between the original and new models lies in the treatment of the ion flux. In the original model, the electric current is assumed to consist of only the electron flux, whereas the contribution of the ion flux is added to the electric current in the new model.

We optimized this particle code for the auroral MMI simulation code. Furthermore, by examining the particle code, we obtained a result similar to that of a previous study, indicating that the code is valid. Also, simulations in the electron drift velocity region, where the original model is not applicable, showed that double layer creation by ionacoustic instability also occurs in the vicinity of $v_{\mathrm{d}} \sim v_{\mathrm{Te}}$. Furthermore, the double layer produced by the Bunemann instability in the case of $v_{\mathrm{d}} \gtrsim 2.0 v_{\mathrm{Te}}$ can be reproduced by the constant current generator model in an open boundary system.

\section{Acknowledgments}

The authors thank Drs. T. Sugiyama and K. Kusano for their valuable discussions. The simulation was carried out on the Earth Simulator/JAMSTEC. This work is performed with the support and under the auspices of the NIFS Collaboration Research program (NIFS07KDAN001).

[1] T. Sato and H. Okuda, Phys. Rev. Lett. 44, 740 (1980).

[2] T. Sato, H. Takamaru and the Complexity Simulation Group, Phys. Plasmas 2, 3609 (1995).

[3] H. Takamaru et al., J. Phys. Soc. Jpn. 66, 3826 (1997).

[4] S. Ishiguro, T. Sato, H. Takamaru and the Complexity Simulation Group, Phys. Rev. Lett. 78, 4761 (1997).

[5] S. Ishiguro et al., Phys. Plasmas 4, 2886 (1997).

[6] R.D. Albert and P.J. Lindstrom, Science 170, 1398 (1970).

[7] F.S. Mozer et al., Phys. Rev. Lett. 38, 292 (1977).

[8] A.J. Hull, J.W. Bonnell, F.S. Mozer and J.D. Scudder, J. Geophys. Res. 108, 1007 (2003).

[9] R.E. Ergun et al., J. Geophys. Res. 109, A12220 (2004).

[10] T. Sato, H. Hasegawa and N. Ohno, Comput. Sci. Disc. 2, 015007 (2009)

[11] T. Sato, J. Phys.: Conf. Ser. 16, 310 (2005).

[12] Message Passing Interface Forum, Int. J. Supercomput. Appl. High Perform. Comput. 8, 159 (1994).

[13] M. Matsumoto and T. Nishimura, ACM Trans. Model. Comput. Simul. 8, 3 (1998).

[14] S. Satake, M. Okamoto, N. Nakajima and H. Takamaru, Lect. Not. Comput. Sci. 4759, 344 (2008).

[15] R.R. Vondrak, H.R. Anderson and R.J. Spiger, J. Geophys. Res. 76, 7701 (1971).

[16] P.A. Cloutier et al., J. Geophys. Res. 78, 640 (1973).

[17] F.W. Berko, R.A. Hoffman, R.K. Burton and R.E. Holzer, J. Geophys. Res. 80, 37 (1975).

[18] P.M. Pazich and H.R. Anderson, J. Geophys. Res. 80, 2152 (1975). 